\section{The zinc-finger proto-oncogene $G$ fi-1b is essential for development of the erythroid and megakaryocytic lineages}

\author{
Shireen Saleque, ${ }^{1}$ Scott Cameron, ${ }^{1-3}$ \\ and Stuart H. Orkin ${ }^{1,4}$
}

\begin{abstract}
${ }^{1}$ Department of Pediatric Oncology, Children's Hospital, Dana Farber Cancer Institute, Harvard Medical School, and Howard Hughes Medical Institute, Boston, Massachusetts 02115, USA; ${ }^{2}$ Department of Biology, Massachusetts Institute of Technology, and Howard Hughes Medical Institute, Cambridge Massachusetts 02139, USA
\end{abstract}

Gfi-1 and $G f i-1 b$ are novel proto-oncogenes identified by retroviral insertional mutagenesis. By gene targeting, we establish that $G f i-1 b$ is required for the development of two related blood lineages, erythroid and megakaryocytic, in mice. $G \mathrm{fi}^{-1 b^{-/-}}$embryonic stem cells fail to contribute to red cells of adult chimeras. $G$ fi-1 $b^{-/-}$embryos exhibit delayed maturation of primitive erythrocytes and subsequently die with failure to produce definitive enucleated erythrocytes. The fetal liver of mutant mice contains erythroid and megakaryocytic precursors arrested in their development. Myelopoiesis is normal. Therefore, $G f i-1 b$ is an essential transcriptional regulator of erythroid and megakaryocyte development.

Received November 1, 2001; revised version accepted December 6, 2001.

Blood cell formation in vertebrates takes place first in a transient wave in the yolk sac blood islands during the period of primitive (or embryonic) hematopoiesis (embryonic days, E7.5-E11 in the mouse). Definitive (or adult) hematopoiesis, which initially occurs in the fetal liver from $\sim$ E11-E18 and then shifts to the bone marrow, sustains blood formation throughout the life of the individual. Definitive hematopoiesis is the product of hematopoietic stem cells (HSCs) that self-renew and also generate progenitors that variously commit to the individual hematopoietic lineages (i.e., erythroid, megakaryocytic, myeloid, and lymphoid).

Commitment of progenitor cells to specific hematopoietic lineages is controlled in part through the combinatorial action of lineage-restricted and more widely expressed transcription factors (Orkin 2000). Gene targeting experiments have been pivotal in defining in vivo requirements of lineage-restricted factors. Loss of single

[Key Words: Transcription factor; hematopoiesis; development; oncogene]

${ }^{3}$ Present address: Department of Pediatrics and of Molecular Biology and Oncology, University of Texas Southwestern Medical Center at Dallas, Dallas, TX 75390, USA.

${ }^{4}$ Corresponding author.

E-MAIL stuart orkin@dfci.harvard.edu; FAX (617) 738-5922.

Article and publication are at http://www.genesdev.org/cgi/doi/10.1101/ gad.959102. factors may lead to failure of HSC formation or expansion (Porcher et al. 1996; Yamada et al. 1998), or to defects in specific hematopoietic lineages (Pevny et al. 1991; Tsang et al. 1998). Of note, the majority of essential hematopoietic transcription factors identified to date are either genetic targets of chromosomal rearrangements or viral integration events associated with leukemias or lymphomas (Okuda et al. 1996; Gilliland 1998; Rabbitts et al. 1999).

Insertional mutagenesis with Moloney murine leukemia virus in c-myc and pim-1 transgenic mice has lead to the identification of oncogenes capable of collaborating with these transgenes in lymphomagenesis /van Lohuizen et al. 1991). Often novel proto-oncogenes discovered in such experiments turn out to be important regulators of normal developmental processes, for example, Bmi-1, a chromatin regulator, crucial for body patterning and hematopoiesis (van der Lugt et al. 1996). Another viral integration site, designated pal-1, was shown to encode Gfi-1, a gene whose expression was also up-regulated by retroviral insertion in T-cell lymphoma lines that grew in an IL-2-independent manner (hence, Gfi for growth factor independent; Gilks et al. 1993; Schmidt et al. 1996; Scheijen et al. 1997). A closely related gene product, Gfi-1b, was isolated by sequence homology (Tong et al. 1998). Other studies have suggested that Gfi-1 and Gfi-1b may regulate cell death or cell cycle programs in cultured cell lines (Grimes et al. 1996b; Tong et al. 1998) and that both genes are expressed in hematopoietic tissues (Tong et al. 1998). Gfi-1 is weakly oncogenic when expressed in T-lymphoid cells of transgenic mice, and cooperates with c-myc (Schmidt et al. 1998). Similarly, $G f i-1 b$ was found to be a target of proviral integrations in retrovirally induced B cell lymphomas in Eu-myc transgenic, pim $1 / \mathrm{pim} 2$ knockout mice (Tong et al. 1998). Both Gfi-1 and Gfi-1b have six zinc-fingers, bind DNA in a sequence-specific manner, and bear a SNAG transcriptional repression domain (Grimes et al. 1996a; ZweidlerMckay et al. 1996; Tong et al. 1998). Interestingly, homologs of $G f i-1 / 1 b$ in other organisms like Drosophila and Caenorhabditis elegans also perform important developmental functions. In Drosophila, the Gfi-1(b)-like senseless gene is necessary for the development of sensory organs (Nolo et al. 2000). In C. elegans, the Gfi-1(b) ortholog pag-3 controls neuroblast cell fate and the identity of its neuronal progeny (Jia et al. 1996, 1997).

As potential oncogenes and critical regulators in diverse developmental contexts, the $G f i-1 / 1 b$ genes may serve important functions in mammalian development. Through targeted gene disruption of $G f i-1 b$ in mice, we establish that $G f i-1 b$ has an essential role in blood cell development, specifically within the erythroid and megakaryocytic cell lineages.

\section{Results}

\section{Disruption of the Gfi-1b gene is embryonic lethal}

Prior studies revealed $G f i-1 b$ expression in spleen and bone marrow (Grimes et al. 1996b; Tong et al. 1998). By Northern blot analysis we observed high-level Gfi-1b expression in erythroid and megakaryocytic cell lines, lowlevel expression in a myeloid line, $\mathrm{Ml}$, and no detectable 
Saleque et al.

expression in lymphoid cells (data not shown). Our results were consistent with a report describing erythroidrestricted expression of a Gfi-1/1b-like RNA (accession no. Y10898) in chicken (Fuchs et al. 1997).

To investigate the function of $G f i-1 b$ in mouse development and/or hematopoiesis, we disrupted the Gfi-1b gene by homologous recombination in embryonic stem (ES) cells. Exons 2-4 of the gene, including the ATG initiator codon in exon 2 , were replaced with a neo ${ }^{R}$ cassette flanked by loxP sites (Fig. 1). Southern blot analysis of BamHI-digested ES cell DNA with a 3' probe was used to identify recombinants (Fig. 1B). Proper targeting was confirmed by long-range PCR from the $n e o^{R}$ cassette to 5 ' sequences (Fig. 1C). Chimeras were generated and matings were performed as previously described (Tsang et al. 1998). Heterozygous $\left(G f i-1 b^{+/-}\right)$mice appeared nor$\mathrm{mal}$ and were fertile. Given that $G \mathrm{fi}-1 \mathrm{~b}^{+/-}$and wild-type mice and embryos appear to be indistinguishable with respect to all assays that we have performed so far, we henceforth refer to either genotype as control. Upon mating of heterozygotes, however, no liveborn $G f_{i-1 b^{-/}}$ mice have been observed, whereas heterozygotes and wild-type offspring were obtained in a ratio of $2: 1$ (data not shown), indicating embryonic lethality of $G f i-1 b^{-/-}$ embryos.

The phenotypic data presented below were obtained from $G f i-1 b^{-1-}$ embryos retaining the neo ${ }^{R}$ gene in the $G f i-1 b$ locus. However, to rule out phenotypic effects due to the inserted neomycin resistance marker, we generated mice and embryos lacking the $n e o^{R}$ cassette. $G f i-1 b^{+/-(\Delta n e o R)}$ mice and $G f i-1 b^{-/-(\Delta n e o R)}$ embryos were indistinguishable from their $G f i-1 b^{+/-(n e o R+1}$ $G f i-1 b^{-/-(\text {neoR+1 }}$ counterparts (data not shown). This shows that the phenotype of the $G f i-1 b^{-/-}$embryos is not caused by transcriptional interference of the inserted $n e o^{R}$ marker on neighboring genes (Manis et al. 1998).

\section{Gfi-1b ${ }^{-/}$ES cells fail to contribute to adult erythropoiesis}

In view of the embryonic lethality of homozygotes and prominent expression in the erythroid lineage, we ascertained whether $G f i-1 b$ is required in a cell-autonomous fashion for production of adult red cells. To this end, $G f i-1 b^{-/-}$ES cells were obtained by selection of $G f_{i-1} 1 b^{+/-}$ ES cells at increased concentration of G418, and chimeras were generated by injection into wild-type C57Bl/6 blastocysts. Several high-level (60\%-90\%) chimeras were generated as estimated by agouti coat color contribution of the 129Sv ES cells. We used the difference between $\mathrm{C} 57 \mathrm{Bl} / 6$ and $129 \mathrm{~Sv}$-derived hemoglobins to assess the contribution of ES-derived cells to red cells of adult chimeras. No Gfi-1b $b^{-1-}$ ES-derived contribution to the hemoglobin of four adult chimeras was detected (Fig. 2, lanes 1-4), whereas $G f i-1 b^{+/-}$ES cells contributed readily (Fig. 2B, lanes 5,6). These experiments suggest that Gfi-1b is required in a cell-autonomous fashion for red blood cell production.

\section{Gfi-1b ${ }^{-/}$embryos exhibit abnormal primitive erythropoiesis}

By timed matings of $G f i-1 b$ heterozygotes, we determined that $G f_{i-1 b^{-/-}}$embryos die by E15. Gfi-1b $b^{-1-}$ embryos are present at the expected frequency of $25 \%$ at E13.5-E14.5 but were terminal and exhibited hemor-

A
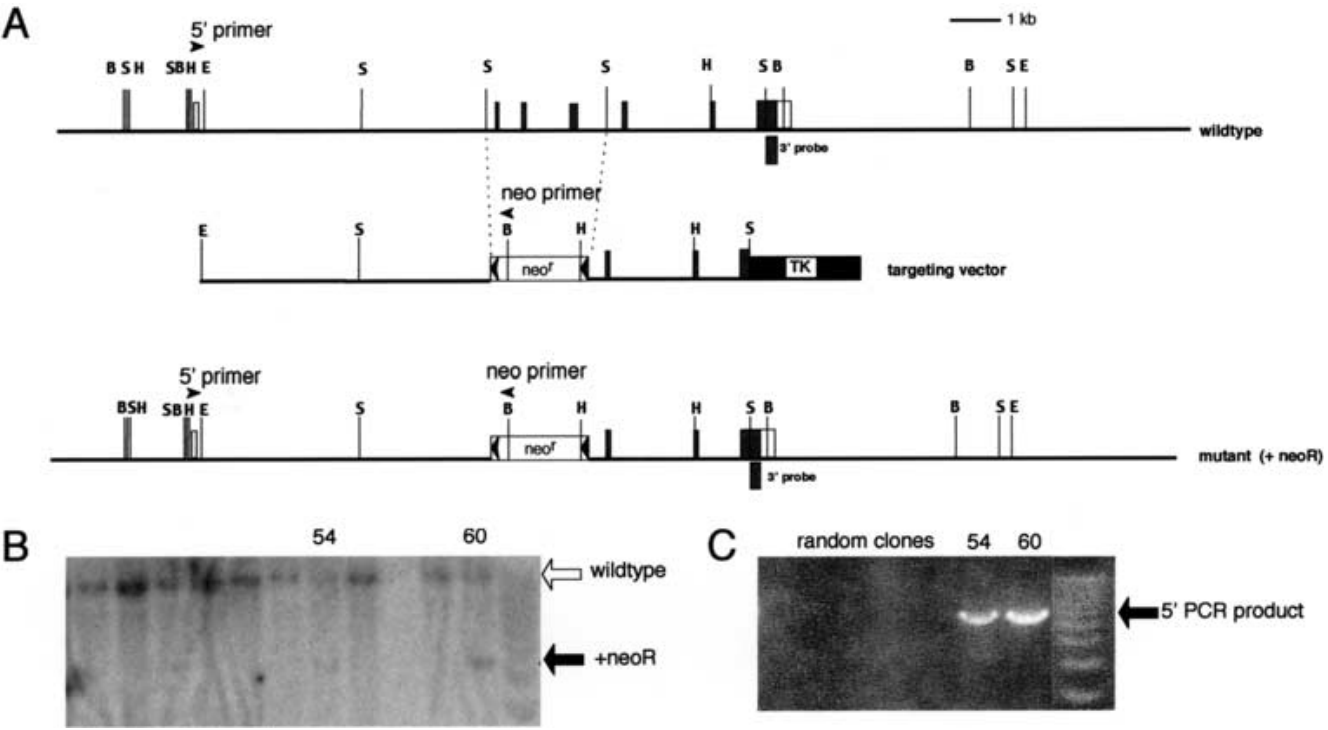

Figure 1. Targeted disruption of the mouse Gfi-1b gene. $(A)$ Partial restriction map of the mouse $G$ fi-1b locus $($ top), the targeting vector (middle), and the expected targeted loci with the floxed neo ${ }^{R}$ cassette (bottom). The 130-bp probe extending from the SacI site to the end of the Gfi-1b coding sequence on exon 7 used to detect appropriate $3^{\prime}$ integration of the targeting vector on Southern blots is indicated ( 3 ' probe). The positions of the primers used to determine the $5^{\prime}$ integration by PCR are also indicated by arrowheads $\left(5^{\prime}\right.$ primer and neo primer, respectively). The Gfi-1b coding exons are indicated as shaded boxes, and the noncoding ones by open boxes. The floxed neo ${ }^{R}$ cassette is indicated by an open box $\left(n e o^{R}\right)$ flanked by arrowheads (lox $P$ sites), and the TK cassette is shown as a solid black box. The restriction enzyme sites indicated in the map are BamHI (B), EcoRI (E), HindIII (H), and SacI (S). The sizes of the BamHI fragment detected by the $3^{\prime}$ probe in the wild-type and the mutant allele with the inserted neo ${ }^{R}$ cassette are $12 \mathrm{~kb}$ and $5 \mathrm{~kb}$, respectively. $(B)$ Southern blot analysis of G148- and gancyclovir-resistant ES cell clones with the $3^{\prime}$ probe. Positions of the wild-type and mutant alleles (with neo ${ }^{R}$ ) are indicated by open and solid arrows, respectively. (C) PCR amplification of selected clones shown in $B$ with the $5^{\prime}$ and neo primers, respectively. The PCR product indicative of the homologous recombination is indicated $\left(5^{\prime}\right.$ PCR product). 


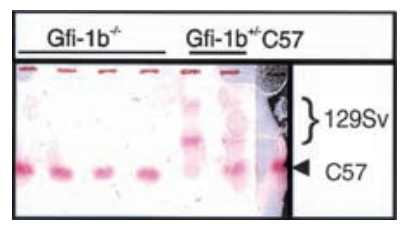

Figure 2. Gfi-1 $1 b^{-/-}$ES cells fail to contribute to adult red cell hemoglobin in chimeric mice. Hemoglobin electrophoresis of peripheral blood from four $G f_{i-1} b^{-/-}$chimeric mice (lanes 1-4), two

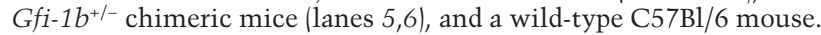

rhage, pallor, and edema suggestive of defects in hematopoiesis (Fig. 3k). Close examination of blood at these and earlier stages revealed abnormal primitive erythropoiesis. Many of the primitive erythrocytes from E9.5E10.5 Gfi-1 $b^{-/-}$embryos exhibit abnormal morphology characterized by extensive membrane blebbing and ruffling (Fig. 3d). In addition, $G f_{i-1 b^{-/}}$primitive erythrocytes are retarded in their overall maturation, as indicated by their less dense nuclei and more basophilic cytoplasm relative to erythrocytes from age-matched control embryos (Fig. 3h,1). These results indicate that $G f i-1 b$ is required for normal primitive erythroid development and that $G f_{i-1 b^{-/-}}$embryos die during the transition from primitive to definitive hematopoiesis.

\section{Gfi-1b is required for definitive erythropoiesis}

By E14.5 the peripheral blood of wild-type and $G f i-1 b^{+/-}$ embryos shows a predominance $(60 \%-70 \%)$ of adult enucleated red blood cells, the product of fetal liver erythropoiesis (Fig. 3j). In contrast, the blood of $G f^{-1}-1 b^{-I-}$ embryos entirely lacks adult red cells (Fig. 31). As this indicates a failure of definitive erythropoiesis, we assessed the stage at which erythropoiesis was arrested in Gfi-1 $b^{-/-}$fetal livers. We performed FACS analysis of fetal liver cells doubly stained with antibodies against ter119, a mouse erythroid-specific marker (Suwabe et al. 1998), and c-kit, a marker of immature hematopoietic cells. Whereas fetal liver cells from wild-type embryos displayed a continuum of cells ranging from $c$-kit $t^{+}$

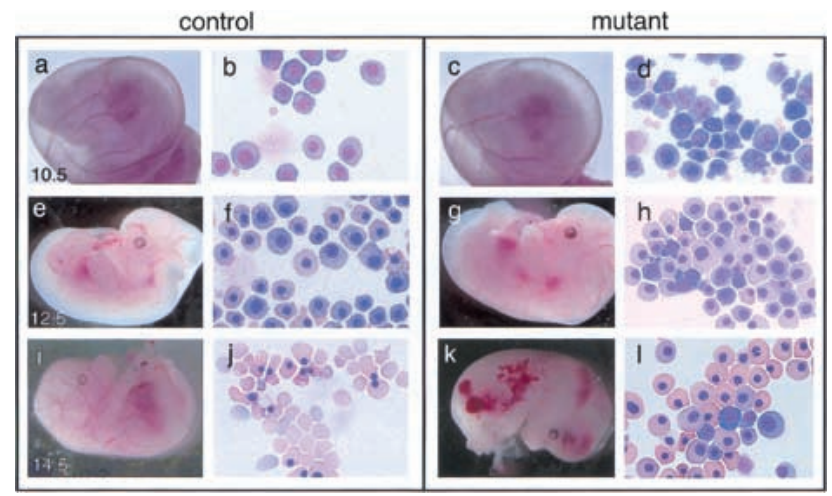

Figure 3. Control and $G f i-1 b$ mutant embryos and peripheral blood at different gestational ages. Control $(a, e, i)$ and mutant $(c, g, k)$ embryos at E10.5, E12.5, and E14.5 and May-Grunwald-Giemsa stains of their corresponding yolk sac blood $(b, f$, and $j$, and $d, h$, and $l$, respectively). Gfi-1 $1 b^{-/-}$embryos show aberrant primitive erythropoiesis characterized by abnormal cell morphology $(d)$ and delayed cellular maturation $(h, 1)$. Embryos die by E15 $(k)$ from a failure of fetal liver erythropoiesis, resulting in the complete absence of definitive enucleated erythrocytes (I).
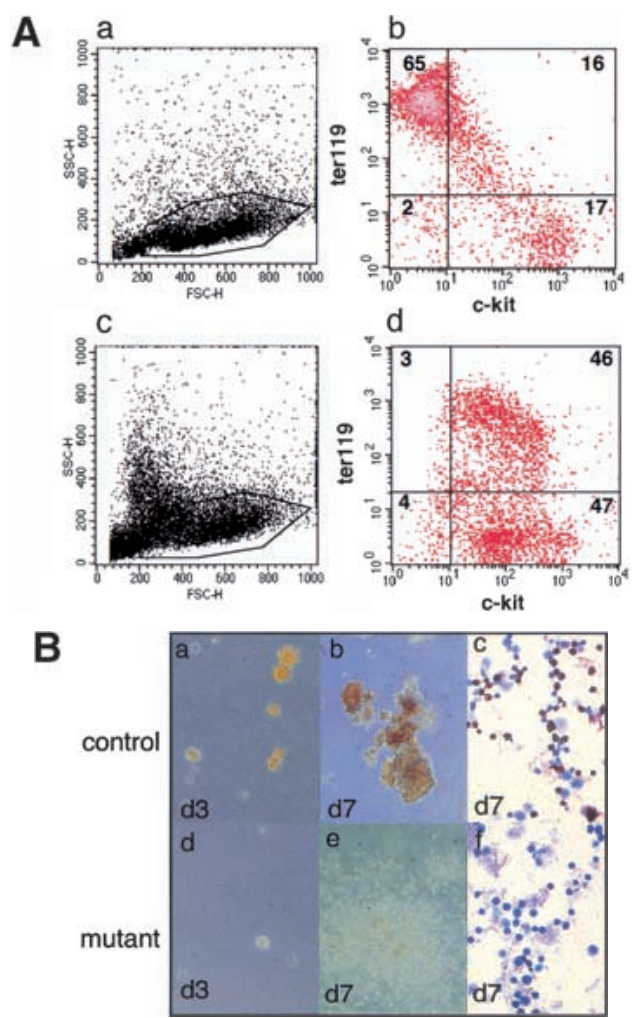

Figure 4. $G f i-1 b^{-/-}$fetal livers show arrested definitive erythropoiesis. (A) Flow cytometry of E12.5 fetal livers. Forward (FSC-H) and side scatter (SSC-H) profiles of control $(a)$ and mutant fetal livers $(c)$. FACS profiles of gated $(b, d)$ fetal liver cells stained with antibodies to $c$-kit and ter 119 . The majority of cells $(60 \%-70 \%)$ from control livers are ter $119^{h i}$ and $c$-kit $(b)$, showing normal erythroid maturation. Cells from $G$ fi-1 $1 b^{-/-}$livers are either ter $119^{-}$or ter $119^{10}$ and $c$-kit ${ }^{+}(d)$. (B) Fetal liver cells from control embryos produce CFU-Es $($ day $3, a)$ and BFU-Es $($ day $7, b)$ when cultured in vitro with epo and KL. Gfi-1 $1 b^{-1-}$ cells proliferate in epo and KL $(e)$ but cannot mature into BFU-Es $(e)$ or CFU-Es $(d)$. Cells from BFU-E colonies of control fetal livers stain positively for benzidine (brown/black cells, $c$ ), but those from $G f i-1 b^{-1-}$ liver colonies do not $(f)$.

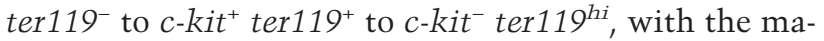
jority of cells $(60 \%-70 \%)$ belonging to the latter category (Fig. 4Ab), fetal livers from Gfi-1 $b^{-/}$embryos contained roughly equal numbers of $c$-kit ter119- and $c$-kit ${ }^{+}$ ter $119^{+}$cells but very few c-kit ter $119^{h i}$ cells (Fig. 4Ad). Hence, Gfi-1 $b^{-/-}$hematopoietic cells commit to the erythroid lineage (based on their expression of ter119), but their further development is blocked, as reflected by continued expression of $c$-kit. Consistent with their immature phenotype, fetal livers from $G f_{i-1 b^{-/-}}$embryos also showed a relative enrichment of $\mathrm{CD} 4^{+}$(another marker of hematopoietic progenitors) cells (data not shown).

To confirm that the developmental arrest observed in the $G \mathrm{fi}-1 b^{-/-}$fetal livers is the consequence of a cellintrinsic defect in the hematopoietic progenitors and is not due to a defective fetal liver environment, we also performed in vitro colony assays with fetal liver cells from E12.5 embryos. When equal numbers of cells were plated in methylcellulose medium supplemented with erythropoietin (epo) and kit ligand (KL), control cells formed many CFU-Es (colony forming units-erythroid) at day 3 or 4 of culture; in contrast, very few, if any, CFU-Es were obtained from $G f i-1 b^{-/-}$embryos at similar 
time points (Fig. 4Bd). The few colonies that arose in cultures from $G f_{i-1 b^{-/-}}$embryos were markedly paler and contained only immature erythroid precursors /data not shown). Beginning at day 4 of culture, rapidly proliferating, dispersed cell colonies were observed in cultures from Gfi-1 $b^{-/-}$embryos. By day 7, these colonies spread over the entire culture dish (Fig. 4Be). These colonies were comprised of arrested erythroid progenitors and mast cells, with the latter cell type predominating as the cultures aged. Cells at this late culture stage (day 7) were stained with benzidine reagent to identify cells with accumulated hemoglobin. Whereas BFU-Es (erythroid burst-forming units) obtained from control livers stained positive (Fig. 4Bc), Gfi-1 $\mathrm{b}^{-/}$cells were negative (Fig. 4Bf). Therefore, the absence of $G f_{i}-1 b$ leads to a developmental arrest of erythroid progenitors in the fetal liver at the BFU-E stage or earlier.

Because $G f i-1 b$ is also expressed in a myeloid cell line, M1 (Tong et al. 1998; data not shown), we evaluated myeloid colony formation of fetal liver cells from $G f i-1 b^{-/-}$mice. Colony assays in the presence of appropriate cytokines revealed equivalent numbers of morphologically normal myeloid cells in the fetal livers of wild-type and Gfi-1 $1 b^{-/-}$mice (data not shown). FACS analysis revealed normal numbers of total $\mathrm{Mac}-1^{+} / \mathrm{Gr}-1^{+}$ cells in E12.5 fetal livers (data not shown). Hence, myeloid development is ostensibly normal in the absence of Gfi-1b.

\section{Gfi-1b is required for megakaryocyte development}

$G f i-1 b$ is highly expressed in megakaryocytic cell lines (data not shown). Fetal liver cells of wild-type and $G \mathrm{fi}-1 b^{+/-}$embryos cultured in methylcellulose media supplemented with the megakaryopoietic cytokine thrombopoietin (tpo) generate abundant colonies containing large, acetylcholine-esterase-positive (a mouse megakaryocyte-specific marker) megakaryocytes (Fig. $5 \mathrm{Aa}-\mathrm{c})$. In marked contrast, $G \mathrm{fi}^{-1 \mathrm{~B}^{-9}}$ fetal liver cells generate colonies containing only small acetylcholineesterase-negative cells (Fig. 5Ad-f). Consistent with the lack of morphologically mature megakaryocytes in Gfi- $1 b^{-/-}$fetal liver cultures, cells from these colonies contained far fewer transcripts for markers of mature megakaryocytes (Fig. 5B), for example, von-Willebrand factor $(\mathrm{vWF})$, transcription factor $\mathrm{p} 45 / \mathrm{NF}-\mathrm{E} 2$, the $c-m p 1$ receptor, and the surface glycoprotein IIb (GpIIb; Shivdasani et al. 1995). Hence, loss of Gfi-1b leads to a block in megakaryopoiesis subsequent to commitment to the megakaryocyte or erythroid/megakaryocytic lineage.

\section{Discussion}

We establish here that the zinc-finger transcription factor $G f i-1 b$ is essential for development of both the erythroid and megakaryocytic cell lineages. Its requirement differs in primitive and definitive erythropoiesis. Primitive erythroid cells in the yolk sac develop in the absence of Gfi-1b, but are morphologically abnormal, characterized by membrane blebbing and delayed cellular maturation. Nonetheless, embryos survive to the fetal liver stage, presumably because of adequate oxygen delivery by these primitive red blood cells. The requirement for Gfi-1b in adult erythropoiesis, however, is more stringent. In its absence, no enucleated erythrocytes are produced, and fetal livers are profoundly deficient in matur-

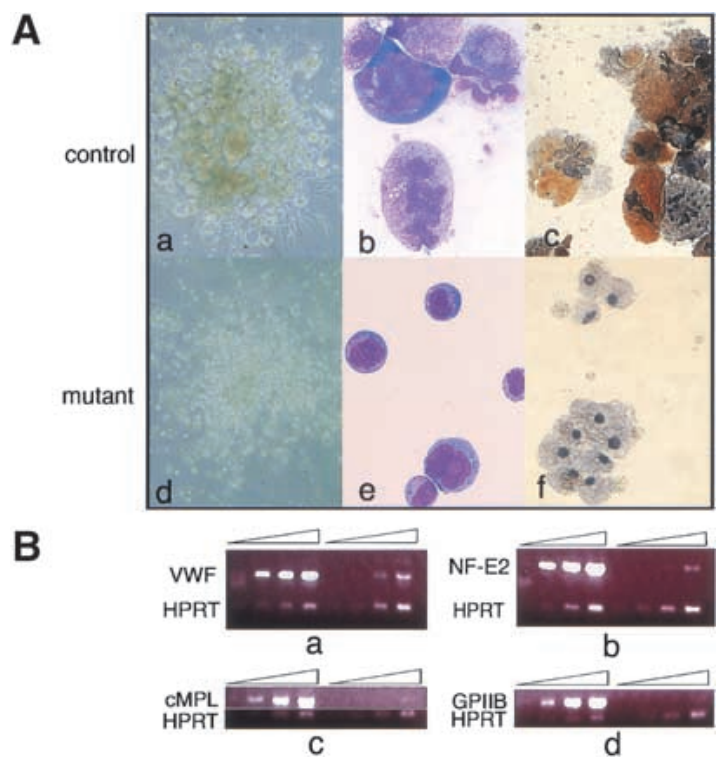

Figure 5. Gfi-1 $b^{-/-}$fetal livers show arrested megakaryopoiesis. $(A)$ Colonies $(a, d)$ and cells $(b, c, e, f)$ from control $(a-c)$ and $G f i-1 b^{-/-}(d-f)$ fetal liver cells grown in thrombopoietin (tpo). When cultured in tpo, fetal liver cells from wild-type livers give colonies with large megakaryocytes $(a)$, whereas $G f i-1 b^{-/-}$cells proliferate in tpo but do not differentiate into large megakaryocytes $(d)$. The $G f_{i-1 b^{-/}}$cells also do not show any nuclear (multilobulation) or cytoplasmic (granulation) maturation upon May-Grunwald-Giemsa staining ( $b$ vs. $e)$ and are negative for acetylcholine esterase staining $(c$ vs. $f)$. $(B)$ Semiquantitative RT-PCR of control (wild-type) and $G \mathrm{fi}^{-1 b^{-/-}}$fetal liver cells cultured in tpo. $G \mathrm{fi}-1 \mathrm{~b}^{-/-}$cells have far fewer transcripts encoding markers of mature megakaryocytes relative to controls ( $a-d$, lanes 5-8 vs. 1-4), for example, von-Willebrand factor (vWF in $a)$, the transcription factor NF-E2 $(b)$, the c-MPL receptor $(c)$, and the surface glycoprotein $\mathrm{IIb}(d)$.

ing erythroid precursors. Embryos, therefore, succumb to anemia during the fetal liver stage of development. Differentiation of megakaryocytes, which are derived from a bipotential erythroid/megakaryocytic progenitor /Orkin $2000)$, is also arrested in the absence of Gfi-1b. In the absence of $G f i-1 b$, presumptive megakaryocytic precursors proliferate in the presence of thrombopoietin, but fail to mature further. This suggests that $G f i-1 b$ is required at a point after commitment to the megakaryocyte lineage. Myeloid development in the absence of $G f i-1 b$ appears normal. Hence, Gfi-1b joins GATA-1 and $F O G-1$, as transcription factors essential for development of the closely related erythroid and megakaryocytic lineages (Pevny et al. 1991; Tsang et al. 1998).

\section{Gfi-1b in the hierarchy of erythroid/megakaryocytic development}

The combined failure of erythroid and megakaryocytic development in $G f_{i-1 b^{-/-}}$embryos is reminiscent of the loss of GATA-1 or FOG-1, factors that act in concert to program differentiation of these lineages. The relationship, if any, of $G f i-1 b$ to the regulatory network controlled by GATA-1/FOG-1 is unknown but worth considering. Gfi-1b might act upstream, downstream, or together with GATA-1/FOG-1 in transcription. We have not observed a significant change in GATA-1 or FOG-1 transcript levels in colonies derived from $G f_{i-1 b^{-/}}$fetal liver cells cultured in erythropoietin and kit ligand (data not shown). Conversely, Gfi-1b is expressed in a 
GATA-1- erythroid cell line (G1E; Weiss et al. 1997; data not shown). Although these findings suggest that the proteins are not dependent on each other for expression, they do not preclude important functional interactions.

Little is known regarding the mechanism of action of Gfi-1b. Although its binding site has been defined by in vitro PCR selection assays, in vivo gene targets remain unknown. Previously, Gfi-1b has been proposed to repress the $p 21^{\text {cip1waf1 }}(p 21)$ gene through a binding site in its promoter region (Tong et al. 1998). The relevance of this observation to hematopoietic development in vivo is uncertain. We have not detected a significant change in p21 RNA levels in Gfi-1b ${ }^{-/-}$fetal liver colonies (data not shown).

$G f i-1 b$ has also been described as a transcriptional repressor based on the presence of a SNAG domain and in vitro reporter assays (Zweidler-Mckay et al. 1996). However, definitive experiments regarding the function of the SNAG repression domain remain to be performed. Ectopic expression of $G f_{i-1} 1 b$ in $\mathrm{CD}_{3} 4^{+}$human progenitor cells augments erythroid cell maturation, and this effect is not dependent on the presence of the SNAG domain (A. Iwama, pers. comm.). Hence, repression of transcription mediated by the SNAG domains may not be the only, or indeed the principal, mode of action of $G f i-1 b$ in erythroid development.

\section{Gfi-1-related proteins as developmental regulators}

The mammalian protein $G f i-1 b$, like its orthologs in Drosophila and C. elegans, Senseless and PAG-3 respectively, regulates the development of specific cellular lineages (Jia et al. 1996, 1997; Nolo et al. 2000). The three proteins also show similar DNA-binding specificities consistent with $>80 \%$ sequence identity between their DNA-binding zinc fingers, and presumably have similar target sites in vivo. However, whether they regulate analogous target genes and pathways in vivo remains to be elucidated. Notably, both PAG-3 and Senseless lack the SNAG repression domain, and this structural variation could lead to mechanistic differences between them in regulating their targets. The control of sensory organ development in Drosophila by an autoregulatory loop comprised of senseless and the basic-helix-loop-helix (bHLH) proneural genes daughterless, achaete-scute, and atonal (Nolo et al. 2000) raises the possibility that $G f i-1 b$ may also interact in a transcriptional network with bHLH factors, within or outside the hematopoietic system. A likely candidate within the hematopoietic system is the bHLH factor SCL/tal-1, a gene required for development of all hematopoietic lineages (Porcher et al. 1996). Because loss of Gfi-1b did not affect SCL/tal-1 expression in fetal liver colonies (data not shown), we conclude that $G f i-1 b$ is not required for $S C L$ expression. Whether SCL regulates $G f i-1 b$ expression is unknown.

Interestingly, Drosophila counterparts of GATA-1 and $F O G$, pannier and u-shaped, respectively, also play unique roles in the formation of a subset of sensory organs, the sensory hair bristles. They do so by reciprocally regulating the genes for achaete and scute (Cubadda et al. 1997; Haenlin et al. 1997). u-shaped and another Drosophila GATA homolog serpent also function coordinately in hemocyte (a primitive hematopoietic cell in flies) development (Fossett et al. 2001), although so far neither senseless nor other Gfi-1 homologs have been implicated in this lineage. Important parallels may therefore exist between Drosophila sensory organ and hemocyte development and mammalian hematopoiesis that merit further investigation.

In conclusion, we have established that the zinc-finger transcription factor $G f i-1 b$, a proto-oncogene able to cooperate with other oncogenes in lymphomagenesis, is essential for the differentiation of the definitive erythroid and megakaryocytic lineages. Its requirement raises important questions regarding the position of Gfi-1b within the hierarchy of hematopoietic transcription factors, and particularly its functional relationship to GATA-1 and FOG-1, previously identified critical regulatory components in these related lineages.

\section{Materials and methods}

Targeted disruption of the murine Gfi-1b gene $G$ fi-1b genomic clones were isolated from a $\lambda$ FixII mouse strain $129 \mathrm{~Sv}$ library (Stratagene). Exon/intron structure was determined by restriction enzyme mapping, PCR, and DNA sequencing. The targeting construct (Fig. 2A) contained 5.5-kb 5' and 2.8-kb 3' homology segments flanking a floxed neo ${ }^{\mathrm{R}}$ cassette and a thymidine kinase gene in the vector pLNTK (Gao et al. 1998). The construct was linearized with PvuI and electroporated into CJ7 mouse ES cells. Transfectants were selected in G418 (280 $\mu \mathrm{g} / \mathrm{mL})$ and gancyclovir $(2 \mu \mathrm{M})$ and expanded for Southern blot analysis and PCR. The targeting frequency was $\sim 2 \%$. A targeted clone was injected into $\mathrm{C} 57 \mathrm{Bl} / 6$ blastocysts to generate chimeras for germ-line transmission.

Generation of Gfi-1b-/- ES cell and chimera analysis $\mathrm{Gfi}^{-1 b^{+/-}}$ES cells were passaged on gelatin-treated plates (Weiss et al. $1994)$ and selected at elevated concentrations $(1-2.5 \mathrm{mg} / \mathrm{mL})$ of G418 (Mortensen et al. 1992). Clones viable at concentrations of 1.9-2.0 mg/ $\mathrm{mL}$ of G418 were subjected to Southern blot analysis to identify homozygous mutants. Hemoglobin analysis of chimeras was performed as previously described (Pevny et al. 1991).

Histology and cytology

Cytocentrifuge preparations were stained with May-Grunwald-Giemsa for general morphology. Benzidine staining and acetylcholine esterase assays were performed by standard methods (Tsang et al. 1998).

\section{In vitro hematopoietic colony assays}

E10.5-E12.5 fetal livers were dissected under sterile conditions. Cells were disaggregated (Wong et al. 1986) and plated in methylcellulose media containing $30 \%$ FCS (Stem cell Technologies), supplemented with one or more of the following cytokines: Epo $(2 \mathrm{U} / \mathrm{mL})$, rat $\mathrm{KL}(50 \mathrm{ng} / \mathrm{mL})$, recombinant human Tpo (1\% v/v of a cell culture supernatant; Villeval et al. 1997).

\section{Flow cytometry}

E12.5 fetal liver cells were disaggregated, stained with fluorochromelabeled antibodies, and scanned in a FACScalibur flow cytometer (Becton Dickinson). The presence of appropriate markers was examined either in the entire cell population or in subpopulations gated according to their forward scatter (size) or side scatter (granularity).

\section{Semiquantitative RT-PCR}

Total RNA was prepared from pooled fetal liver colonies using the RNeasy kit (QIAGEN), then 100 ng of total RNA was used as the template in a one-step RT-PCR reaction (QIAGEN) with previously described megakaryocyte-specific and HPRT primers (Tsang et al. 1998). Aliquots were withdrawn at 24, 26, 28, and 30 cycles of PCR and examined on an agarose gel.

\section{Acknowledgments}

We thank Carol Browne for invaluable assistance in ES cell culture and Yuko Fujiwara, Aimee Williams, and Shelly Galusha for generation of chimeric mice. S.S. is a Research Associate of the HHMI. S.H.O. is an Investigator of the HHMI.

The publication costs of this article were defrayed in part by payment 
of page charges. This article must therefore be hereby marked "advertisement" in accordance with 18 USC section 1734 solely to indicate this fact.

\section{References}

Cubadda, Y., Heitzler, P., Ray, R.P., Bourouis, M., Ramain, P., Gelbart, W., Simpson, P., and Haenlin, M. 1997. u-shaped encodes a zinc finger protein that regulates the proneural genes achaete and scute during the formation of bristles in Drosophila. Genes \& Dev. 11: 3083-3095.

Fossett, N., Tevosian, S.G., Gajewski, K., Zhang, Q., Orkin, S.H., and Schulz, R.A. 2001. The Friend of GATA proteins U-shaped, FOG-1, and FOG-2 function as negative regulators of blood, heart, and eye development in Drosophila. Proc. Natl. Acad. Sci. 98: 7342-7347.

Fuchs, B., Wagner, T., Rossel, N., Antoine, M., Beug, H., and Niessing, J. 1997. Structure and erythroid cell-restricted expression of a chicken cDNA encoding a novel zinc finger protein of the Cys + His class. Gene 195: 277-284.

Gao, Y., Sun, Y., Frank, K.M., Dikkes, P., Fujiwara, Y., Seidl, K.J., Sekiguchi, J.M., Rathbun, G.A., Swat, W., Wang, J., et al. 1998. A critical role for DNA end-joining proteins in both lymphogenesis and neurogenesis. Cell 95: 891-902.

Gilks, C.B., Bear, S.E., Grimes, H.L., and Tsichlis, P.N. 1993. Progression of interleukin-2 (IL-2)-dependent rat T cell lymphoma lines to IL-2independent growth following activation of a gene (Gfi-1) encoding a novel zinc finger protein. Mol. Cell. Biol. 13: 1759-1768.

Gilliland, D.G. 1998. Molecular genetics of human leukemia. Leukemia 12 Suppl 1: S7-S12.

Grimes, H.L., Chan, T.O., Zweidler-McKay, P.A., Tong, B., and Tsichlis, P.N. 1996a. The Gfi-1 proto-oncoprotein contains a novel transcriptional repressor domain, SNAG, and inhibits G1 arrest induced by interleukin-2 withdrawal. Mol. Cell. Biol. 16: 6263-6272.

Grimes, H.L., Gilks, C.B., Chan, T.O., Porter, S., and Tsichlis, P.N 1996b. The Gfi-1 protooncoprotein represses Bax expression and inhibits T-cell death. Proc. Nat1. Acad. Sci. 93: 14569-14573.

Haenlin, M., Cubadda, Y., Blondeau, F., Heitzler, P., Lutz, Y., Simpson, P., and Ramain, P. 1997. Transcriptional activity of pannier is regulated negatively by heterodimerization of the GATA DNA-binding domain with a cofactor encoded by the u-shaped gene of Drosophila. Genes \& Dev. 11: 3096-3108

Jia, Y., Xie, G., and Aamodt, E. 1996. pag-3, a Caenorhabditis elegans gene involved in touch neuron gene expression and coordinated movement. Genetics 142: 141-147.

Jia, Y., Xie, G., McDermott, J.B., and Aamodt, E. 1997. The C. elegans gene pag-3 is homologous to the zinc finger proto-oncogene gfi-1. Development 124: 2063-2073.

Manis, J.P., van der Stoep, N., Tian, M., Ferrini, R., Davidson, L., Bottaro, and Alt, F.W. 1998. Class switching in B cells lacking $3^{\prime}$ immunoglobulin heavy chain enhancers. J. Exp. Med. 188: 1421-1431.

Mortensen, R.M., Conner, D.A., Chao, S., Geisterfer-Lowrance, A.A., and Seidman, J.G. 1992. Production of homozygous mutant ES cells with a single targeting construct. Mol. Cell. Biol. 12: 2391-2395.

Nolo, R., Abbott, L.A., and Bellen, H.J. 2000. Senseless, a Zn finger transcription factor, is necessary and sufficient for sensory organ development in Drosophila. Cell 102: 349-362.

Okuda, T., van Deursen, J., Hiebert, S.W., Grosveld, G., and Downing, J.R. 1996. AML1, the target of multiple chromosomal translocations in human leukemia, is essential for normal fetal liver hematopoiesis. Cell 84: 321-330.

Orkin, S.H. 2000. Diversification of haematopoietic stem cells to specific lineages. Nat. Rev. Genet. 1: 57-64.

Pevny, L., Simon, M.C., Robertson, E., Klein, W.H., Tsai, S.F., D'Agati, V., Orkin, S.H., and Costantini, F. 1991. Erythroid differentiation in chimaeric mice blocked by a targeted mutation in the gene for transcription factor GATA-1. Nature 349: 257-260

Porcher, C., Swat, W., Rockwell, K., Fujiwara, Y., Alt, F.W., and Orkin, S.H. 1996. The T cell leukemia oncoprotein SCL/tal-1 is essential for development of all hematopoietic lineages. Cell 86: 47-57.

Rabbitts, T.H., Bucher, K., Chung, G., Grutz, G., Warren, A., and Yamada, Y. 1999. The effect of chromosomal translocations in acute leukemias: The LMO2 paradigm in transcription and development. Cancer Res. 59: 1794s-1798s.
Scheijen, B., Jonkers, J., Acton, D., and Berns, A. 1997. Characterization of pal-1, a common proviral insertion site in murine leukemia virusinduced lymphomas of c-myc and Pim-1 transgenic mice. J. Virology 71: 9-16.

Schmidt, T., Zornig, M., Beneke, R., and Moroy, T. 1996. MoMuLV proviral integrations identified by Sup-F selection in tumors from in fected myc/pim bitransgenic mice correlate with activation of the gfi-1 gene. Nucleic Acids Res. 24: 2528-2534.

Schmidt, T., Karsunky, H., Gau, E., Zevnik, B., Elsasser, H.P., and Moroy, T. 1998. Zinc finger protein GFI-1 has low oncogenic potential but cooperates strongly with pim and myc genes in T-cell lymphomagenesis. Oncogene 17: 2661-2667.

Shivdasani, R.A., Rosenblatt, M.F., Zucker-Franklin, D., Jackson, C.W., Hunt, P., Saris, C.J., and Orkin, S.H. 1995. Transcription factor NF-E2 is required for platelet formation independent of the actions of thrombopoietin/MGDF in megakaryocyte development. Cell 81: 695-704.

Suwabe, N., Takahashi, S., Nakano, T., and Yamamoto, M. 1998. GATA-1 regulates growth and differentiation of definitive erythroid lineage cells during in vitro ES cell differentiation. Blood 92: 4108-4118.

Tong, B., Grimes, H.L., Yang, T.Y., Bear, S.E., Qin, Z., Du, K., El-Deiry, W.S., and Tsichlis, P.N. 1998. The Gfi-1B proto-oncoprotein represses p21WAF1 and inhibits myeloid cell differentiation. Mol. Cell. Biol. 18: $2462-2473$.

Tsang, A.P., Fujiwara, Y., Hom, D.B., and Orkin, S.H. 1998. Failure of megakaryopoiesis and arrested erythropoiesis in mice lacking the GATA-1 transcriptional cofactor FOG. Genes \& Dev. 12: 1176-1188.

van der Lugt, N.M., Alkema, M., Berns, A., and Deschamps, J. 1996. The Polycomb-group homolog Bmi-1 is a regulator of murine Hox gene expression. Mech. Dev. 58: 153-164.

van Lohuizen, M., Verbeek, S., Scheijen, B., Wientjens, E., van der Gulden, H., and Berns, A. 1991. Identification of cooperating oncogenes in $\mathrm{E} \mu-\mathrm{myc}$ transgenic mice by provirus tagging. Cell 65: 737-752.

Villeval, J.L., Cohen-Solal, K., Tulliez, M., Giraudier, S., Guichard, J., Burstein, S.A., Cramer, E.M., Vainchenker, W., and Wendling, F. 1997. High thrombopoietin production by hematopoietic cells induces a fatal myeloproliferative syndrome in mice. Blood 90: 4369-4383.

Weiss, M.J., Keller, G., and Orkin, S.H. 1994. Novel insights into erythroid development revealed through in vitro differentiation of GATA-1 embryonic stem cells. Genes \& Dev. 8: 1184-1197.

Weiss, M.J., Yu, C., and Orkin, S.H. 1997. Erythroid-cell-specific properties of transcription factor GATA-1 revealed by phenotypic rescue of a gene-targeted cell line. Mol. Cell. Biol. 17: 1642-1651.

Wong, P.M., Chung, S.W., Chui, D.H., and Eaves, C.J. 1986. Properties of the earliest clonogenic hemopoietic precursors to appear in the developing murine yolk sac. Proc. Natl. Acad. Sci. 83: 3851-3854.

Yamada, Y., Warren, A.J., Dobson, C., Forster, A., Pannell, R., and Rabbitts, T.H. 1998. The T cell leukemia LIM protein Lmo2 is necessary for adult mouse hematopoiesis. Proc. Nat1. Acad. Sci. 95: 3890-3895.

Zweidler-Mckay, P.A., Grimes, H.L., Flubacher, M.M., and Tsichlis, P.N. 1996. Gfi-1 encodes a nuclear zinc finger protein that binds DNA and functions as a transcriptional repressor. Mol. Cell. Biol. 16: 40244034 . 


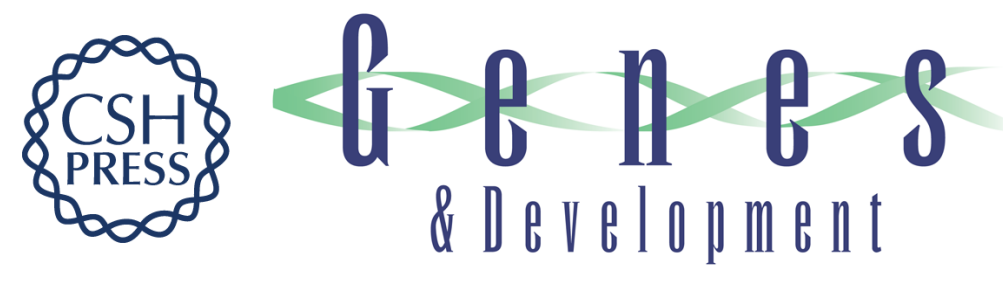

\section{The zinc-finger proto-oncogene Gfi-1b is essential for development of the erythroid and megakaryocytic lineages}

Shireen Saleque, Scott Cameron and Stuart H. Orkin

Genes Dev. 2002, 16:

Access the most recent version at doi:10.1101/gad.959102

References This article cites 32 articles, 20 of which can be accessed free at: http://genesdev.cshlp.org/content/16/3/301.full.htmI\#ref-list-1

License

Email Alerting

Receive free email alerts when new articles cite this article - sign up in the box at the top Service right corner of the article or click here.

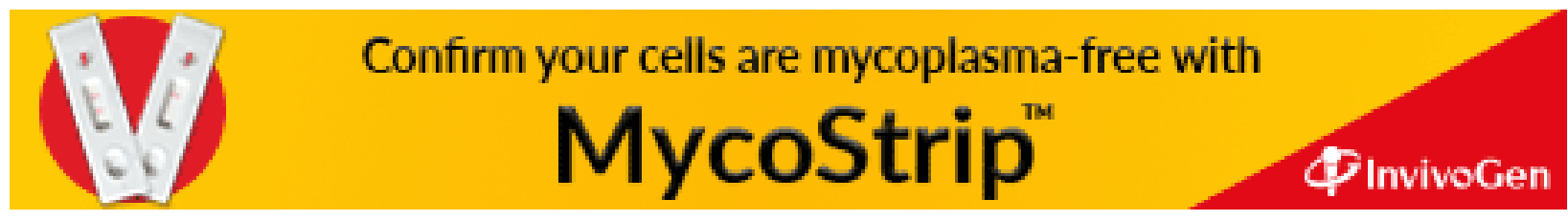

